J Urol Nephrol

June 2015 Vol.:2, Issue:1

(c) All rights are reserved by Kapoor et al.

\section{Secondary Vesical Calculi Formation on Non Absorbable Sutures Following Abdominal Hysterectomy: A Case Report}

Keywords: la trogenic; Vesic al calc uli; Cystolitholapaxy; Secondary vesic al calculus; Hysterec tomy

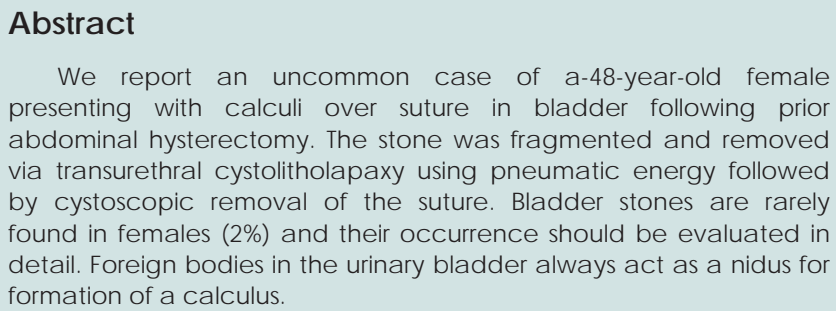

We report an uncommon case of a-48-year-old female presenting with calculi over suture in bladder following prior abdominal hysterectomy. The stone was fragmented and removed via transurethral cystolitholapaxy using pneumatic energy followed by cystoscopic removal of the suture. Bladder stones are rarely found in females (2\%) and their occurrence should be evaluated in detail. Foreign bodies in the uninary bladder always act as a nidus for formation of a calculus.

\section{Introduction}

Bladder (vesical) calculi are stones or calcified materials that are present in the bladder (or in a bladder substitute that functions as a urinary reservoir). Bladder outlet obstruction remains the most common cause of bladder calculi in adults. In males, prostatic enlargement, elevation of the bladder neck, and high post void residual urine volume leads to secondary calculi due to urinary stasis and recurrent urinary tract infections. Foreign bodies with contact to urine can cause urinary stones. Self-manipulation or iatrogenic causes lead to foreign bodies in the bladder. Examples of iatrogenic causes include suture material, clips, catheters, ureteral stents or migration of an intrauterine device as well as unrecognised perforations occurring due to erosion of urinary incontinence meshes [1].

Bladder stones are rarely found in females (2\%) and their occurrence should be evaluated in detail [2]. Most bladder calculi secondary to female pelvic surgery result from either obstruction or foreign objects [3]. The presence of suture material in the bladder is a well-known cause of urinary tract infection and stone formation [4]. We hereby report an uncommon case of a 48-year-old female presenting with calculus over suture in bladder following prior abdominal hysterectomy.

\section{Case Report}

A 48- year- old female presented with severe lower urinary tract symptoms (LUTS) since 6 months comprising of frequency, dysuria, lower abdominal pain and occasional haematuria. She was initially treated for UTI (urinary tract infection) by the local physician. She had undergone abdominal hysterectomy for fibroid uterus and menorrhagia 4 years back. Urine examination showed pyuria and microscopic haematuria and urine culture showed E.coli. Ultrasonography revealed a normally distended bladder with thickened walls measuring $5 \mathrm{~mm}$ and two vesical calcului of 1.57 $\mathrm{cm}$ and $1.2 \mathrm{~cm}$ size respectively (Figure 1). Cystoscopy revealed

\section{Journal of}

Urology \& Nephrology

\author{
Deepa Kapoor ${ }^{1 *}$, Vijayendra S. Kanwar², Bernard \\ Amer $^{2}$, Khumukcham $S^{2}$, Ajoijam Kaku Singh ${ }^{2}$ and \\ Rajendra Singh Sinam ${ }^{2}$ \\ ${ }^{1}$ Department of Obstetrics \& Gynaecology, Bhimrao Ambedker \\ Hospital, New Delhi, India \\ ${ }^{2}$ Department of Urology, Regional Institute of Medical Sciences, \\ Imphal, India \\ Address for Correspondence \\ Deepa Kapoor, Senior Resident, Department of Obstetrics \& \\ Gynaecology, Bhimrao Ambedker Hospital, New Delhi, India, E-mail: \\ deepa.kapoor@rediffmail.com \\ Submission: 02 June 2015 \\ Accepted: 15 June 2015 \\ Published: 19 June 2014 \\ Reviewed \& Approved by: Dr. Lampros P. Mitrakas, Scientific \\ Associate of Urology Department, University of Thessaly, University \\ Hospital of Larissa, Greece
}

two calculi which were fixed to an underlying suture (Figure 2) and arising from the base of bladder. The stone was fragmented using Swiss Master Lithoclast pneumatic energy and removed. During the procedure it was noted that the calculi were attached to two prolene sutures that were used for the vault closure during hysterectomy (Figure 3). Cystoscopic scissors were used to cut the sutures and release them off the bladder wall, followed by forceps extraction of

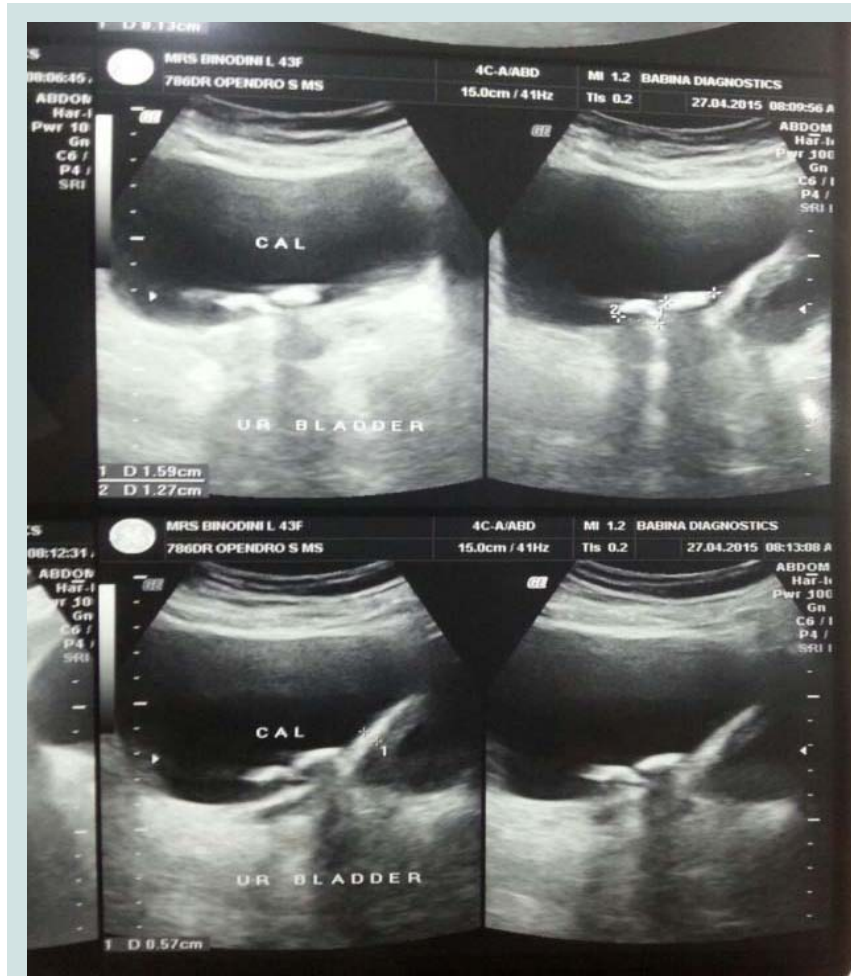

Figure 1: Transabdominal ultrasound showing vesical calculi. 
Citation: Kapoor D, Kanwar VS, Amer B, Khumukcham S, Singh AK, et al. Secondary Vesical Calculi Formation on Non Absorbable Sutures Following Abdominal Hysterectomy: A Case Report. J Urol Nephrol. 2015;2(1): 3.

the excised sutures. Cystogram with instillation of saline mixed with diluted methylene blue dye in bladder did not reveal any leakage per vaginum. Vaginoscopy did not reveal any fistula formation or suture material in vault. Patient's post operative course was uneventful. On follow up at 1 month, the patient was asymptomatic and urine culture was sterile. Cystoscopy revealed that the site of suture had completely healed.

\section{Discussion}

As reported in the literature, the presence of foreign bodies in the bladder is rare with only a very few small case series reporting both self-introduced and iatrogenic introduction of foreign bodies into the bladder (IBI: Iatrogenic bladder injury) [5]. IBT can be broadly divided into external and internal bladder injuries. The former type is associated with pelvic procedures and mostly occurs during obstetric and gynaecologic procedures, followed by general surgical and urologic interventions [5,6]. Internal bladder injuries mainly occur during cystoscopic procedures, namely transurethral resection of bladder tumours (TURBT) where bladder wall perforations can occur. There are two types of bladder wall perforations, intra- and extra-peritoneal, with the former being more common and requiring intervention [1]. Iatrogenic foreign bodies inside the bladder can be caused by dislodgment or breaking of the intravesical instruments, forgotten surgical gauze, non absorbable sutures, or staples used in pelvic procedures. In addition, unrecognized perforations can occur due to erosion of urinary incontinence meshes [7] and migration of IUCD (intrauterine contraceptive devices).

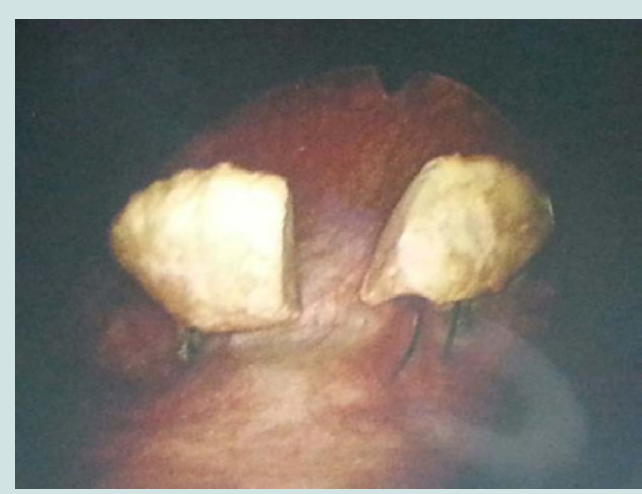

Figure 2: Cystoscopy showing inflamed bladder wall with two vesical calculi fixed and formed over the suture.

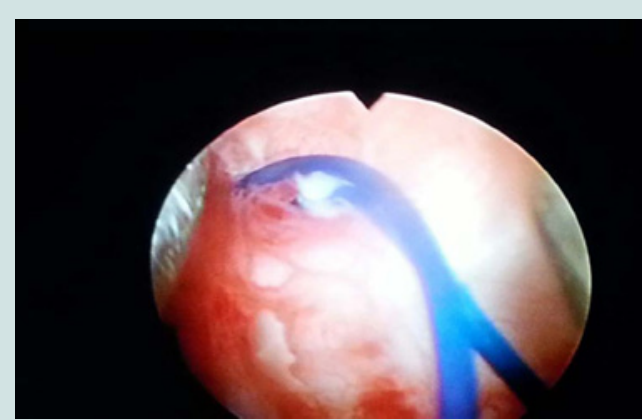

Figure 3: The non-absorbable suture prolene seen in the bladder wall.
The literature reports that direct injuries, are usually recognised intra-operatively and the indirect injuries tends to have a delayed presentation, mimicking bladder neoplasms in some instances $[1,8]$. This may one of a few cases reported on vesical stones secondary to the presence of prolene sutures in the bladder wall $[1,8,9]$. Similar studies have been reported following TAH (total abdominal hysterectomy), Inguinal hernia repair and orthopedic surgeries [10-12].

The non absorbable suture we found in the bladder was probably the result of inadvertent placement in the bladder base while suturing the vault or subsequent erosion of a loop of suture in the bladder lumen, creating a nidus for stone formation [3]. The fundamental inciting event of calculus development is crystal formation: nucleation occurs around a foreign object as a result of infection or secondary to obstruction with resulting super saturation and then aggregation, proliferation for crystal growth, and eventual stone formation [2].

The most important principle in treating bladder calculi is eradication of the underlying cause, such as relieving obstruction and eliminating infection. Accurate diagnosis is clearly paramount. Sonography successfully identified a retained suture as the likely cause. As the retained suture served as a nidus for crystallization, its contour was well demarcated on sonography. A number of techniques are available to remove bladder stones. The use of cystoscopic scissors for the release of sutures in the bladder is a safe and effective technique in the management of foreign bodies in the bladder.

This case demonstrates the importance of good surgical technique and exposure during operative procedures, a preferable use of absorbable suture for closure of vaginal vault and that significant lower urinary tract symptoms (with or without recurrent UTI) in women with a history of pelvic surgery demand a through cystoscopic examination to rule out inadvertent intravesical suture placement $[3,13]$.

\section{Conclusion}

The incidence of vesical calculi is on a rise in women due to an increase in pelvic procedures and surgery for incontinence [11]. Any female presenting with LUTS and recurrent UTI with previous surgical history should be thoroughly evaluated and the physician should keep a high index of suspicion for vesical calculi. Vesical calculi can rarely be caused after abdominal hysterectomy. We present a rare case of bladder stones secondary to an iatrogenic injury to the bladder in the form of prolene sutures protruding into the bladder following an abdominal hysterectomy. The use of cystoscopic scissors for the release of sutures in the bladder is a safe and effective technique in the management of foreign bodies in the bladder.

\section{References}

1. Rafique M (2008) Intravesical foreign bodies: review and current management strategies. Urol J 5: 223-231.

2. Drach GW (1992) Urinary lithiasis: etiology, diagnosis, and medical treatment In: Walsh RF, Retik AB, Stamey TA, Vaughan ED (eds). Campbell's Urology. 6th ed. Philadelphia, PA: WB Saunders Co; 2085-2156.

3. Schwartz BF, Stoller ML (2000) The vesical calculus. Urol Clin North Am 27 333-346.

4. Trevino R, Goldstein AM, Vartanian NL (1979) Vesical bladder stones forming around non-absorbable sutures and possible explanation for their delayed appearance. J Urol 122: 849. 
Citation: Kapoor D, Kanwar VS, Amer B, Khumukcham S, Singh AK, et al. Secondary Vesical Calculi Formation on Non Absorbable Sutures Following Abdominal Hysterectomy: A Case Report. J Urol Nephrol. 2015;2(1): 3.

\section{ISSN: $2380-0585$}

5. Summerton D, Kitrey N, Lumen N, Serafetinidis E, Djakovic N (2012) EAU Guidelines on iatrogenic trauma. Eur Urol 62: 628-639.

6. Dobrowolski F, Linczinsky W, Drewniak T, Jakubik P, Kusionowicz J (2002) External and iatrogenic trauma to the bladder: a survey in Poland. BJU Int 89 755-756.

7. Al-Awadi KA, Zaghloul AS, Kehinde EO (2011) Symptomatic secondary vesical calculus formed on an intrauterine contraceptive device inserted 25 years previously. Urol Int 86: 483-486.

8. Frenkl TL, Rackley RR, Vasavada SP, Goldman HB (2008) Management of iatrogenic foreign bodies of the bladder and urethra following pelvic floor surgery. Neurourol Urodyn 27: 491-495.

9. Kehinde EO, Ali Y, Al-Hunayan A, Al-Awadi KA, Mahmoud AH (2000)
Complications associated with using non-absorbable sutures for ureteroneocystostomy in renal transplant operations. Transplant Proc 32: 1917-1918.

10. Chawla A, Mishra DK, Kumar SP (2014) Secondary vesical calculus in female. Austin J Urol 1: 2

11. Almarzouq A, Mahmoud AH, Ashebu SD, Kehinde EO (2014) Vesical calculus formation on non-absorbable sutures used for open inguinal hernia repair. Int J Surg Case Rep 5: 811-815.

12. Rub R, Madeb R, Morgenstern S, Ben-Chaim J, Avidor Y (2001) Development of a large bladder calculus on sutures used for pubic bone closure following exstrophy repair. World J Urol 19: 261-262.

13. van Ophoven A, deKernion JB (2000) Clinical management of foreign bodies of the genitourinary tract. J Urol 164: 274-287. 